\title{
Effect of detrusor botulinum toxin a injection on urothelial dysfunction in patients with chronic spinal cord injury: a clinical and immunohistochemistry study before and after treatment
}

\author{
S-F Chen, C-H Chang and H-C Kuo
}

Objective: The objective of this study is to investigate the changes of urothelial junction proteins, apoptosis and suburothelial inflammation after detrusor injection of botulinum toxin A (BoNT-A) in patients with spinal cord injury (SCl) and neurogenic detrusor overactivity (NDO).

Methods: A total of 26 patients with chronic suprasacral SCI and NDO were enroled. The urothelium was assessed by cystoscopic biopsy at baseline, 3 and 6 months after a single treatment of $300 \mathrm{U}$ BoNT-A into the detrusor. Immunofluorescence staining of E-cadherin, zonula occludens-1 (ZO-1) and tryptase for mast cell activity were performed. Urothelial apoptosis was also evaluated. The differences in urothelial dysfunction were compared between baseline and 3 and 6 months after treatment. Bladder biopsies from patients undergoing anti-incontinence surgery served as controls.

Results: A single 300-U BoNT-A injection into the detrusor significantly decreased detrusor pressure and increased bladder compliance at 3 and 6 months after treatment. Significantly lower E-cadherin and ZO-1 expressions and increased mast cell and apoptotic cell counts were noted in $\mathrm{SCl}$ bladders compared with controls (all $P<0.001$ ). Significantly greater distributions of E-cadherin $(P<0.001)$ and ZO-1 $(P=0.05)$ expressions were noted 3 months after BoNT-A injection. However, these changes had declined by 6 months after treatment. Activated mast cells and urothelial apoptosis showed no significant differences between baseline and 3 or 6 months.

Conclusion: Urothelial dysfunction and adhesive and junction protein concentrations in SCI patients' bladders recovered after BoNT-A treatment. However, this effect decreased with time. Thus, neurogenic inflammation after $\mathrm{SCl}$ was not adequately improved after a single BoNT-A injection.

Spinal Cord (2016) 54, 889-894; doi:10.1038/sc.2015.241; published online 2 February 2016

\section{INTRODUCTION}

Spinal cord injury (SCI) is a significant cause of morbidity and mortality in developing countries, with a global annual incidence of 1:25 000. ${ }^{1}$ Neurogenic detrusor overactivity (NDO) is a consequence of the change in bladder reflex pathways in chronic SCI patients. Neurogenic voiding dysfunction and urinary symptoms in patients with SCI lead to several complications. If not well managed, high intravesical pressure damages the upper urinary tract, causing renal scarring and chronic renal insufficiency, which impair the quality of life. ${ }^{2}$ SCI also leads to rapid disruption of the uroepithelial barrier. This manifests in a loss of cell-cell interactions, decreases in transepithelial resistance and increases in water and urea permeability. ${ }^{3}$

We have found decreased expression of urothelial adhesion and junction proteins and increased suburothelial inflammation and apoptosis in patients with chronic SCI, regardless of injury level. ${ }^{4}$ Disruption of the urothelial barrier initiates a cascade of bladder dysfunction events, leading to suburothelial inflammation and vulnerability to chronic or recurrent cystitis or infection, or both.
Suburothelial inflammation might also affect urothelial function, leading to a vicious cycle. Botulinum toxin A (BoNT-A) has been useful in the treatment of NDO in SCI patients. ${ }^{5}$ It is possible that the decrease of bladder inflammation might also improve the urothelial barrier function after detrusor BoNT-A injection.

BoNT-A is one of the most powerful neurotoxins; it inhibits the release of neurotransmitters from the nerve fibres and urothelium. ${ }^{6}$ It may be possible to stem the disruption of the uroepithelium by modulating the release of neurotransmitters and inflammatory mediators. BoNT-A injection decreases bladder nerve growth factor production and urinary nerve growth factor levels, suggesting an anti-inflammatory response can be achieved after intravesical BoNT-A treatment. ${ }^{7}$ Besides, our previous study reported BoNT-A treatment improved urothelial dysfunction in interstitial cystitis and overactive bladder. ${ }^{8,9}$

Until now, there is insufficient data to determine definitively whether or not BoNT-A injection into the detrusor improves the urothelial dysfunction in patients with SCI and NDO. The present preliminary study explored the mechanisms of action of BoNT-A 
treatment on urothelial dysfunction in patients with chronic SCI and NDO. We investigated the immunohistochemistry characteristics of bladders from SCI patients at baseline and after BoNT-A treatment by analysing adhesion and junction proteins, inflammation and apoptotic signalling molecules after one single BoNT-A injection treatment.

\section{PATIENTS AND METHODS}

In this preliminary, prospective study, 26 patients with chronic SCI and NDO, who were refractory to antimuscarinic treatment were consecutively enroled into the study. Video-urodynamic studies were routinely performed before enrolment, to confirm the presence of detrusor overactivity (DO) with or without detrusor sphincter dyssynergia (DSD). Patients were excluded if they had an active urinary tract infection (UTI) or urinary tract cancer at enrolment, history of lower urinary tract surgery, or chronic systemic diseases such as congestive heart failure and chronic renal failure. All patients had been educated to perform clean intermittent catheterization (CIC) due to possible urinary retention after BoNT-A injection. The study was approved by the Institutional Review Board and Ethics Committee of Buddhist Tzu Chi General Hospital. Each patient was informed about the study rationale and procedures, and written informed consent to participate in the study was obtained before any bladder procedure.

Each patient received $300 \mathrm{U}$ of BoNT-A injection into the detrusor muscle under intravenous general anaesthesia in the operating room. Each vial of BoNT-A (100 U) was diluted with $10 \mathrm{ml}$ of normal saline and 30 injections evenly distributed into detrusor were given using a 23-gauge needle and a rigid cystoscopic injection instrument (22 Fr, Richard Wolf, Knittlingen, Germany). The bladder cold cup biopsies were performed randomly at the posterior wall above the interureteric ridge and only the bladder mucosa and submucosa were taken to prevent bladder perforation.

We also invited 10 patients with stress urinary incontinence, but without urgency frequency symptoms, to serve as controls for comparison. All patients had been proven free of bladder outlet obstruction (BOO) by videourodynamic study. Male patient was not selected to serve as the controls, because the prostate might cause $\mathrm{BOO}$, which may affect the urothelial function. ${ }^{10}$ The $\mathrm{BOO}$ in women was diagnosed by radiologic evidence of bladder outlet narrowing plus a voiding detrusor pressure $>35 \mathrm{~cm} \mathrm{H}_{2} \mathrm{O}$ and a maximum flow rate $<15 \mathrm{ml} \mathrm{s}^{-1}$, or a voiding detrusor pressure $>40 \mathrm{~cm}$ $\mathrm{H}_{2} \mathrm{O} .{ }^{11}$ In male patients, $\mathrm{BOO}$ was defined by the Abrams Griffiths number (Pdet.Qmax $-2 \times$ Qmax). An Abrams Griffiths number $>40$ was defined as having BOO. ${ }^{12}$ For SCI patients, a BOO was defined if there was radiological evidence of bladder outlet narrowing during voiding cystourethrography, regardless of the voiding pressure. The bladder biopsy specimens were sent to the pathology department, to exclude the possibility of carcinoma in situ. At 3 and 6 months after the BoNT-A treatment, patients underwent another bladder biopsy. Specimens were stored frozen in liquid nitrogen for further investigation.

Immunofluorescence staining and quantification of protein expression in the bladder tissues of SCI patients and controls were investigated for urothelial adhesion and junction proteins by measuring E-cadherin expression and zonula occludens-1 (ZO-1) expression, respectively. Mast cell activation was assessed by measuring tryptase levels. Urothelial cell apoptosis was evaluated using the TUNEL (TdT dUTP nick end labelling) assay as described previously. ${ }^{13,14}$

The urinary bladder specimens were immersed and fixed for $1 \mathrm{~h}$ in an icecold solution of $4 \%$ formaldehyde in phosphate-buffered saline ( $\mathrm{pH}$ 7.4). They were then rinsed with ice-cold phosphate-buffered saline containing $15 \%$ sucrose for $12 \mathrm{~h}$. Biopsy specimens were embedded in optimum cutting temperature medium and stored at $-80^{\circ} \mathrm{C}$. Four sections per specimen were cut using a cryostat at a thickness of $8 \mu \mathrm{m}$ and collected on new silane III-coated glass slides (Muto Pure Chemicals Co. Ltd, Tokyo, Japan). The expression of E-cadherin and ZO-1 in the urothelium was quantified using Image J software, developed by the National Institutes of Health (Bethesda, MD). ${ }^{15}$ Immunofluorescence (tryptase and TUNEL assays) was quantified by counting the number of positively stained cells/total cells per unit area $\left(4 \mu \mathrm{m}^{2}\right)$. The results are shown as the percentage of positive cells per 100 total cells. Differences in expressions of the proteins in the urothelium at baseline and 3 and 6 months after the BoNT-A injection were analysed using the paired $t$-test. All calculations were performed using SPSS for Windows, version 16.0 (SPSS, Chicago, IL, USA). $P<0.05$ was considered to indicate statistical significance.

\section{RESULTS}

A total of 26 patients with chronic SCI and 10 controls were enroled in the study. The patients included 17 men and 9 women with a mean age of $42.7 \pm 13.1$ years. Among them, 20 had complete injuries, while 6 had incomplete injuries. The injury level was at cervical spinal cord in 12 patients and the thoracic cord in 14. Among them, 10 cervical SCI and 8 thoracic SCI patients had DSD. About the bladder management of SCI patients, 16 voided spontaneously or by reflex, while 5 used percussion to void, 3 voided by abdominal pressure and 2 used CIC. No patient having indwelling catheters or cystostomy was enroled in this study. The control group included 10 women with stress urinary incontinence with a mean age of $51.4 \pm 12.1$ years.

No severe side effects such as general weakness or severe autonomic dysreflexia occurred after BoNT-A injection. Five patients had slight hematuria, which was spontaneously resolved in 3 days without intervention. UTI was carefully monitored during the follow-up visits and antibiotic treatment was given in six $(23 \%)$ patient who had symptomatic or asymptomatic UTI. Seven (27\%) patients developed urinary retention and needed CIC. In patients with quadriplegia, who could not perform CIC by himself, we taught the family or caregiver to perform CIC, to prevent autonomic dysreflexia.

Table 1 shows the clinical characteristics of the SCI patients at baseline and 3 and 6 months after treatment with $300 \mathrm{U}$ BoNT-A detrusor injection. Compared with the baseline data, the detrusor pressure significantly decreased at 3 and 6 months after BoNT-A injection $\left(17.1 \pm 20.4\right.$ and $23.6 \pm 23.1 \mathrm{~cm} \mathrm{H}_{2} \mathrm{O}$, respectively, versus $35.7 \pm 23.3 \mathrm{~cm} \mathrm{H} \mathrm{H}_{2} \mathrm{O}$, all $\left.\mathrm{P}<0.05\right)$. Bladder compliance showed a significant increase $\left(48.2 \pm 46.5\right.$ and $55.9 \pm 78.3 \mathrm{ml} \mathrm{cm} \mathrm{H}_{2} \mathrm{O}^{-1}$ versus $41.7 \pm 54.8 \mathrm{ml} \mathrm{cm} \mathrm{H}_{2} \mathrm{O}^{-1}$, all $\left.\mathrm{P}<0.05\right)$. The post-void residual volume also significantly increased after treatment $(375.0 \pm 157.4$ and $312.2 \pm 177.6 \mathrm{ml}$, respectively, versus $229.6 \pm 138.7 \mathrm{ml}$, all $P<0.05)$. However, the cystometric bladder capacity, glomerular filtration rate and serum creatinine levels did not change significantly after BoNT-A

Table 1 Clinical features of the spinal cord-injured patients at baseline, 3 months and 6 months after BoNT-A injection

\begin{tabular}{|c|c|c|c|}
\hline & Baseline & 3 Months & 6 Months \\
\hline Cystometric bladder capacity (ml) & $289.8(255.6)$ & $\begin{array}{c}422.0(131.5) \\
P=0.156\end{array}$ & $\begin{array}{c}361.1(152.81) \\
P=0.269\end{array}$ \\
\hline Detrusor pressure $\left(\mathrm{cmH}_{2} \mathrm{O}\right)$ & $35.7(23.3)$ & $\begin{array}{c}17.1(20.4) \\
P=0.001\end{array}$ & $\begin{array}{c}23.6(23.1) \\
P=0.06\end{array}$ \\
\hline $\mathrm{Qmax}\left(\mathrm{ml} \mathrm{s}^{-1}\right)$ & $3.92(4.75)$ & $\begin{array}{c}2.92(5.14) \\
P=0.499\end{array}$ & $\begin{array}{c}3.71(5.71) \\
P=0.906\end{array}$ \\
\hline PVR (ml) & $229.6(138.7)$ & $\begin{array}{c}375.0(157.4) \\
P<0.0001\end{array}$ & $\begin{array}{c}312.2(177.6) \\
P=0.033\end{array}$ \\
\hline Compliance & $41.7(54.8)$ & $\begin{array}{c}48.2(46.5) \\
P=0.017\end{array}$ & $\begin{array}{c}55.9(78.3) \\
P=0.003\end{array}$ \\
\hline Detrusor overactivity & $24 / 26$ & $14 / 26$ & $16 / 26$ \\
\hline Glomerular filtration rate $(\mathrm{ml} \mathrm{min}-1)$ & $84.17(19.97)$ & $\begin{array}{c}84.68(24.17) \\
P=0.836\end{array}$ & $\begin{array}{c}81.96(23.81) \\
P=0.553\end{array}$ \\
\hline Serum creatinine $\left(\mathrm{mg} \mathrm{dl}^{-1}\right)$ & $0.72(0.16)$ & $\begin{array}{c}0.77(0.24) \\
P=0.183\end{array}$ & $\begin{array}{c}0.77(0.22) \\
P=0.007\end{array}$ \\
\hline
\end{tabular}

Abbreviations: BoNT-A, botulinum toxin A; PVR, post-void residual volume; Qmax, maximum flow rate.

Data are expressed as mean (s.d.). P-values indicate difference between baseline and time points. 
Table 2 Changes in bladder expression of E-cadherin, activated mast cells, apoptosis (TUNEL) and ZO-1 in the controls and SCI patients after a single BoNT-A injection at 3 and 6 months compared with baseline

\begin{tabular}{|c|c|c|c|c|}
\hline & Control $(\mathrm{N}=10)$ & SCl_Baseline $(\mathrm{N}=26)$ & SCl_3M $(\mathrm{N}=26)$ & $S C I \_6 M(\mathrm{~N}=26)$ \\
\hline E-cadherin & $42.4 \pm 16.73$ & $31.27 \pm 19.84^{a}$ & $\begin{array}{c}52.88 \pm 19.55^{a} \\
P<0.001\end{array}$ & $\begin{array}{c}43.64 \pm 19.88^{a} \\
P=0.07\end{array}$ \\
\hline Mast cell & $1.25 \pm 1.15$ & $16.46 \pm 5.9^{a}$ & $\begin{array}{c}16.1 \pm 4.64^{a} \\
P=0.75\end{array}$ & $\begin{array}{c}14.48 \pm 4.21^{\mathrm{a}} \\
P=0.30\end{array}$ \\
\hline TUNEL & $0.08 \pm 0.26$ & $3.6 \pm 2.14^{a}$ & $\begin{array}{c}4.05 \pm 2.16^{\mathrm{a}} \\
P=0.16\end{array}$ & $\begin{array}{c}4.58 \pm 3.03^{a} \\
P=0.06\end{array}$ \\
\hline ZO-1 & $11.01 \pm 5.66$ & $6.50 \pm 6.35^{a}$ & $\begin{array}{c}8.65 \pm 3.86^{a} \\
P=0.05\end{array}$ & $\begin{array}{c}7.3 \pm 4.44^{a} \\
P=0.55\end{array}$ \\
\hline
\end{tabular}

Abbreviations: BoNT-A, botulinum toxin A; SCI, spinal cord injured; TUNEL, TdT dUTP nick end labelling; ZO-1, zonula occludens-1.

$P$-values indicate difference between baseline and time points.

aSignificantly different from the control; data are expressed as mean \pm s.d.

treatment. The therapeutic effectiveness showed no significant difference between patients with different SCI level or with and without DSD, or patients with and without performing CIC.

At baseline, expression of E-cadherin, ZO-1, apoptosis and mast cell activity in the patients differed significantly compared with the control group (Table 2). A significant increase in mast cell infiltration was observed in the bladder specimens from SCI patients $(P<0.001)$. The expressions of E-cadherin and ZO-1 in the bladders of SCI patients were significantly lower compared with the corresponding expressions in the control bladders $(P<0.001)$. TUNEL staining showed significantly higher numbers of apoptotic cells in SCI bladder tissue compared with the control bladder tissue $(3.6 \pm 2.14$ versus $0.08 \pm 0.26$ cells per 100 total cells, $P<0.001)$.

At 3 months after BoNT-A injection, a significantly higher distribution of E-cadherin expression compared with the baseline was observed $(52.88 \pm 19.55$ versus $31.27 \pm 19.84$ fluorescence intensity units per $\left.4 \mu \mathrm{m}^{2}, P<0.001\right)$. At 6 months after treatment, the distribution was $43.64 \pm 19.88$ versus $31.27 \pm 19.84, \quad P=0.07$. A significantly higher distribution of $\mathrm{ZO}-1 \quad(8.65 \pm 3.86$ versus $6.50 \pm 6.35$ cells per 100 total cells, $P=0.05$ ) at 3 months after BoNT-A injection compared with the baseline level was also noted. However, ZO-1 expression was not significantly increased at 6 months compared with baseline $(7.3 \pm 4.44$ cells per 100 total cells, $P=0.55)$. The mast cell activity showed no significant difference between baseline and 3 months $(16.46 \pm 5.9$ versus $16.1 \pm 4.64$ cells per 100 total cells, $P=0.75$ ), nor did urothelial cell apoptosis differ significantly at 3 months after BoNT-A treatment compared with baseline $(4.05 \pm 2.16$ versus $3.6 \pm 2.14$ cells per 100 total cells, $P=0.16)$. At 6 months after BoNT-A injection, the changes in expression of E-cadherin, mast cell activation, apoptosis and ZO-1 did not differ compared with baseline. Nevertheless, all bladder parameters of SCI patients at 3 and 6 months significantly differed from the controls. The changes of urothelial dysfunction parameters showed no significant difference between different level SCI patients or between patients with and without DSD.

Figure 1 shows the expression of ZO-1, E-cadherin, mast cell counts and apoptotic cell counts (TUNEL staining) in bladder tissues from SCI patients at baseline and 3 and 6 months after a single injection of $300 \mathrm{U}$ of BoNT-A.

\section{DISCUSSION}

The results of this study revealed impaired expression of the adhesion protein E-cadherin and tight junction protein $\mathrm{ZO}-1$, and significantly higher mast cell activation and cell apoptosis in the urothelium of patients with chronic SCI and NDO. Although detrusor pressure and compliance were improved after a single BoNT-A injection, only E-cadherin and ZO-1 expressions were increased at 3 months. However, these changes were not significant at 6 months after treatment, indicating that the effect of single BoNT-A injection on urothelial dysfunction is short term.

Low urinary tract dysfunction and complications are major concerns in the management of SCI patients. Although C-fibre afferents have a role in the development of hyperactivity in NDO due to SCI, there is evidence for the involvement of the urothelium in the development and symptoms of NDO. ${ }^{16}$

Apodaca et al. ${ }^{3}$ found that SCI also led to rapid disruption of the uroepithelial barrier. This was manifested in loss of cell-cell interactions, decreases in transepithelial resistance and increases in water and urea permeability. ${ }^{3}$ One of the significant changes observed is the occurrence of disruption of the urothelium accompanied by reduction in transepithelial resistance. This happened as early as $2 \mathrm{~h}$ after SCI, but some recovery of transepithelial resistance occurred at later time points. ${ }^{3}$ In addition, changes in the morphology of the urothelium have been detected, including the disappearance of apical cells, disorganization of cell layers and reduction of cellular volume. ${ }^{3}$ Studies have shown that the expression of the purinergic receptor P2X2 in the human urothelium of SCI patients is increased. ${ }^{17}$ In addition, rats with SCI also had increased release of excitatory mediators including prostaglandins, adenosine triphosphate and nitric oxide. ${ }^{18,19}$ These mediators could contribute to increased excitability of bladder sensory afferents present in the suburothelial region or among urothelial cells. Another study also revealed that nerve growth factor mRNA and protein levels are increased in the urinary bladders of rats with SCI. ${ }^{20} \mathrm{~A}$ number of ion channels including transient receptor potential vanilloid receptor 1 also increase in patients with neurogenic bladder disease. ${ }^{21}$ Another major consequence of SCI is increased expression of connexin 26 in the urothelium, which could have a role in DO. ${ }^{22}$ These evidence show the importance of urothelial dysfunction, which induces inflammation and deteriorates in a vicious cycle.

Studies of animals with SCI revealed that factors released from the mucosal bladder layer influence spontaneous detrusor contractions. ${ }^{23}$ This activity may involve a number of factors including release of adenosine triphosphate from the urothelium acting on the underlying cells within the suburothelial space. In addition, chronic SCI in animals (and in patients with urge symptoms) shows an increase in connexin-hemichannel expression and increased intercellular communication within the low urinary tract compared with healthy control groups. ${ }^{24,25}$ These findings suggest that altered low urinary tract junction proteins, perhaps by contributing to the amplification of 

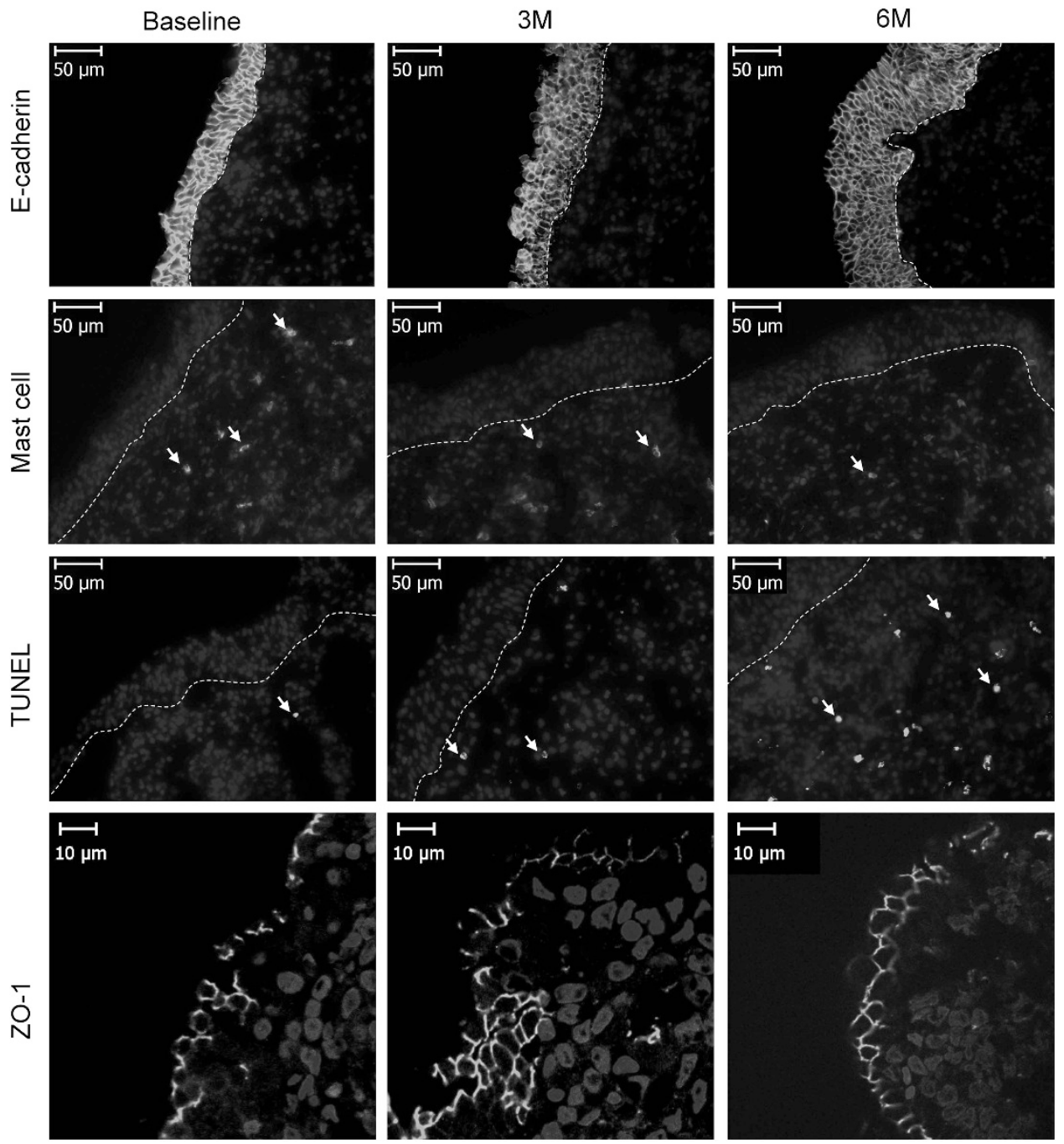

Figure 1 E-cadherin expression, mast cell counts, apoptotic cell counts (TUNEL staining) and ZO-1 expression in bladders of patients with SCls at baseline and 3 and 6 months after a single 300-U BoNT-A injection. The target cells are labelled in bright (as the arrows indicate) and the white dotted lines indicate the boundary between the urothelium and suburothelium.

chemical signalling between and within low urinary tract cells, may have a role in the development of DO following SCI.

SCI is associated with a number of changes to the urothelium, including both structural changes in addition to defects in urothelial signalling changes. ${ }^{26}$ Pretreatment with hexamethonium could block the acute effects of SCI on uroepithelial function, indicating that the release of neurotransmitters by bladder efferent nerves is at least partially responsible for the disruption of the uroepithelium. ${ }^{3}$ BoNT-A inhibits adenosine triphosphate release from the urothelial, but not the serosal side of the bladder. This suggests that BoNT-A treatment inhibits transmitter release not only from efferent nerve endings but sensory nerve terminals and the urothelium as well. ${ }^{6}$

E-cadherin is a calcium-dependent glycoprotein, which has a critical role in cell-to-cell adhesion. Loss of E-cadherin function can trigger cancer progression and metastasis. ${ }^{14} \mathrm{ZO}-1$ is a tight junction protein that maintains the highly resistant urothelial barrier. ${ }^{27}$ Previous study has also established a molecular connection between E-cadherin and TRPV4, suggesting that E-cadherin is associated with bladder sensation and barrier function. ${ }^{28}$ In the current study, the results showed the urothelial barrier recovered after BoNT-A injection by improving the adhesion and tight junction protein levels. This could explain the mechanism of decreasing the hyperexcitability of C-fibre bladder afferents, thereby reducing NDO. However, the effect was not sustained over time.

The mast cells degranulate and release pre-formed mediators of inflammation including histamine, heparin and tryptase. ${ }^{29}$ Apoptosis is a stepwise process characterized by a series of stereotypical morphological changes that eventually lead to cell death. Urothelial cell apoptosis in patients with interstitial cystitis/bladder pain syndrome resulted from upregulation of the inflammatory signals, including p38 mitogen-activated protein kinase and tumor necrosis factor- $\alpha .{ }^{30}$ In the present study, we showed that the apoptotic process was highly activated in the bladders of patients with SCI compared with the control group. However, the active mast cell activity and urothelial apoptosis showed no significant difference after single BoNT-A injection. These results indicate that BoNT-A injection cannot have an adequate anti-inflammatory effect in a single injection.

The bladder urothelium has a significant role in the sensory transduction mechanisms modulating micturition, in particular in conditions of increased sensory nerve transmission following chronic inflammation and SCI. Animal studies have shown that BoNT-A decreases transmitter release from the urothelium and neuropeptide 
release from the bladder afferents. ${ }^{22,23}$ In patients with NDO, the increasing nerve growth factor in bladder tissue decreased or normalized with detrusor injection of BoNT-A. ${ }^{31}$ BoNT-A has been shown to inhibit contraction of both smooth and skeletal muscle by blocking neurotransmitter release. In humans with NDO, the bladder muscarinic receptors $\mathrm{M} 2$ and $\mathrm{M} 3$, and purinergic receptors $\mathrm{P} 2 \mathrm{X} 2$ and $\mathrm{P} 2 \mathrm{X} 3$ are reduced after detrusor BoNT-A injections, suggesting that BoNT-A inhibits DO by acting through inhibition of both the sensory and motor arms of the micturition reflex. ${ }^{30}$

Since 2000, Schurch et al. ${ }^{32}$ reported the good effect of BoNT-A toxin detrusor injection for treating NDO in spinal cord-injured patients. Ongoing improvement in urodynamic parameters and incontinence was already present in all patients re-evaluated at 16 and 36 weeks. In addition to the benefit of decreased detrusor pressure to prevent renal damage, it significantly improved quality of life. ${ }^{33}$ In the past 10 years, most of the study used $300 \mathrm{U}$ of BoNT-A, usually 30 injections of $10 \mathrm{U} \mathrm{ml}^{-1}$ in the bladder (excluding the trigone) under cystoscopic guidance and with different types of anaesthesia. ${ }^{34}$ In this study, we used $300 \mathrm{U}$ BoNT-A for treatment of NDO with SCI bladders since 2006. Several phase 3, large-scale, randomized, doubleblind, placebo-controlled, multicentre trials were conducted to investigate the efficacy and safety of detrusor BoNT-A injections in patients with NDO, due to SCI or multiple sclerosis (MS). BoNT-A 200 or $300 \mathrm{U}$ significantly reduced urinary incontinence episodes compared with placebo. The effects were equally observed in both SCI and multiple sclerosis patients, and less side effects occurred in the 200-U treatment group. ${ }^{5}$ Based on these phase 3 trials, the regulatory approved dose of BoNT-A is $200 \mathrm{U}$ for patients with NDO. Haferkamp et al. ${ }^{35}$ reported regeneration of the nerve endings after an interval of about 9 months after BoNT-A detrusor injection, with no significant ultrastructural detrusor changes found in the biopsy tissue. In addition to inhibiting detrusor contraction, there is evidence that the BoNT-A inhibits bladder sensory pathways contributing to storage symptoms. ${ }^{36-38}$

Our previous study revealed that BoNT-A injection decreased detrusor pressure and increase bladder compliance after repeat treatments every 6 months. ${ }^{39}$ The therapeutic effectiveness showed no significant difference between patients with different SCI level or with and without DSD. ${ }^{40}$ Patients with different clinical demographics and urodynamic parameters had similar treatment outcomes and UDI-6 scores. ${ }^{36,39}$ The performance of CIC or not did not affect the satisfactory effect of Botox as well. ${ }^{40}$ According to the results of this study, urothelial barrier dysfunction in SCI patients' bladders recovered after BoNT-A treatment; however, this effect decreased with time. Thus, neurogenic inflammation after SCI was not adequately improved after a single 300-U BoNT-A injection; it seems rational to repeat BoNT-A injection at a 6-month interval if the treatment target is at the urothelial dysfunction. In this study, the result showed the urothelial dysfunction improvement after BoNT-A injection in the expression of the adhesion protein E-cadherin and the tight junction protein ZO-1. Therefore, we supposed that BoNT-A injection improved NDO in SCI bladder not only by anticholinergic effects but urothelium function may be recovered after treatment.

UTI is a possible cause for urothelial dysfunction in patients with or without SCI. Most SCI patients may have asymptomatic bacteriuria and might effect on urothelial dysfunction. There has been no study on this topic, However, the asymptomatic bacteriuria might result in increased excitability of suburothelial sensory fibres and induce DO at a small bladder volume. Our previous study also showed the changes of urothelial function after recurrent UTI was similar to that occuring in this study. ${ }^{41}$ In this study, patients were excluded if they had an active UTI at enrolment. Although six patients developed symptomatic or asymptomatic UTI after BoNT-A injection, the improvement of E-cadherin and ZO-1 are still present at 3 months after BoNT-A injection. However, the early disappearance of BoNT-A effect on the urothelial dysfunction might, in part, be affected by the UTI episodes.

A limitation of the present study is the small case number and lack of a control arm. However, this preliminary study demonstrated the mechanisms of action of BoNT-A treatment to improve urothelial dysfunction in the bladders of patients with chronic SCI. Another limitation is that there is no histologic assessment, which may provide compatible information to support our results. Further investigation of repeated injections and long-term follow-up is ongoing. In addition, the therapeutic effects of sensory protein improvements of transient receptor potential vanilloid receptor $1, \mathrm{P} 2 \mathrm{X}$ receptors, connexin and endothelial nitric oxide synthase have not yet been thoroughly investigated and require further study.

\section{CONCLUSION}

Urothelial dysfunction and adhesive and junction protein concentrations in SCI bladders recovered at 3 months after a single $300-\mathrm{U}$ BoNT-A injection. However, this effect had decreased at 6 months after treatment. Thus, a single BoNT-A injection treatment to the detrusor produced an inadequate therapeutic response without a long lasting impact on chronic inflammation and urothelial apoptosis.

\section{DATA ARCHIVING}

There were no data to deposit.

\section{CONFLICT OF INTEREST}

The authors declare no conflict of interest.

1 Sekhon LH, Fehlings MG. Epidemiology, demographics, and pathophysiology of acute spinal cord injury. Spine 2001; 26: S2-S12.

2 Patki PS, Hamid R, Arumugam K, Shah PJ, Craggs M. Botulinum toxin-type A in the treatment of drug-resistant neurogenic detrusor overactivity secondary to traumatic spinal cord injury. BJU Int 2006; 98: 77-82.

3 Apodaca G, Kiss S, Ruiz W, Meyers S, Zeidel M, Birder L. Disruption of bladder epithelium barrier function after spinal cord injury. Am J Physiol Renal Physiol 2003; 284: F966-F976.

4 Jiang YH, Liu HT, Kuo HC. Urothelial dysfunction and chronic inflammation in patients with spinal cord injuries at different levels and correlation with urodynamic findings. Neurourol Urodyn 2014; 34: 757-762.

5 Ginsberg D, Gousse A, Keppenne V, Sievert KD, Thompson C, Lam W et al. Phase 3 efficacy and tolerability study of onabotulinumtoxinA for urinary incontinence from neurogenic detrusor overactivity. J Urol 2012; 187: 2131-2139.

6 Khera M, Somogyi GT, Kiss S, Boone TB, Smith CP. Botulinum toxin A inhibits ATP release from bladder urothelium after chronic spinal cord injury. Neurochem Int 2004; 45: 987-993.

7 Liu HT, Kuo HC. Intravesical botulinum toxin A injections plus hydrodistension can reduce nerve growth factor production and control bladder pain in interstitial cystitis. Urology 2007; 70: 463-468.

8 Jhang JF, Jiang YH, Kuo HC. Potential therapeutic effect of intravesical botulinum toxin type A on bladder pain syndrome/interstitial cystitis. Int J Urol 2014; 21: 49-55.

9 Kuo HC, Liu HT, Chuang YC, Birder LA, Chancellor MB. Pilot study of liposomeencapsulated onabotulinumtoxina for patients with overactive bladder: a singlecenter study. Eur Urol 2014; 65: 1117-1124.

10 Yuk SM, Shin JH, Song KH, Na YG, Lim JS, Sul CK. Expression of brain derivedneurotrophic factor and granulocyte-colony stimulating factor in the urothelium: relation with voiding function. BMC Urol 2015; 15: 37.

11 Kuo HC. Videourodynamic characteristics and lower urinary tract symptoms of female bladder outlet obstruction. Urology 2005; 66: 1005-1009

12 Griffiths D, Hofner K, van Mastrigt R, Rollema HJ, Spangberg A, Gleason D. Standardization of terminology of lower urinary tract function: pressure-flow studies of voiding, urethral resistance, and urethral obstruction. International Continence Society Subcommittee on Standardization of Terminology of Pressure-Flow Studies. Neurourol Urodyn 1997: 16: 1-18.

13 Shie JH, Liu HT, Wang YS, Kuo HC. Immunohistochemical evidence suggests repeated intravesical application of botulinum toxin $A$ injections may improve treatment efficacy of interstitial cystitis/bladder pain syndrome. BJU Int 2013; 111: 638-646. 
14 Shie JH, Kuo HC. Higher levels of cell apoptosis and abnormal E-cadherin expression in the urothelium are associated with inflammation in patients with interstitial cystitis/ painful bladder syndrome. BJU Int 2011; 108: E136-E141.

15 Kaczmarek E, Gorna A, Majewski P. Techniques of image analysis for quantitative immunohistochemistry. Rocz Akad Med Bialymst 2004; 49: 155-158.

16 Cruz CD, Cruz F. Spinal cord injury and bladder dysfunction: new ideas about an old problem. Scientific World Journal 2011; 11: 214-234.

17 Pannek J, Janek S, Sommerer F, Tannapfel A. Expression of purinergic P2X2-receptors in neurogenic bladder dysfunction due to spinal cord injury: a preliminary immunohistochemical study. Spinal Cord 2009; 47: 561-564.

18 Smith CP, Gangitano DA, Munoz A, Salas NA, Boone TB, Aoki KR et al. Botulinum toxin type $A$ normalizes alterations in urothelial ATP and NO release induced by chronic spinal cord injury. Neurochem Int 2008; 52: 1068-1075.

19 Masunaga K, Yoshida M, Inadome A, Iwashita H, Miyamae K, Ueda S. Prostaglandin E2 release from isolated bladder strips in rats with spinal cord injury. Int J Urol 2006; 13: 271-276.

20 Steers WD, Tuttle JB. Mechanisms of disease: the role of nerve growth factor in the pathophysiology of bladder disorders. Nat Clin Pract Urol 2006; 3: 101-110.

21 Cruz F, Dinis P. Resiniferatoxin and botulinum toxin type A for treatment of lower urinary tract symptoms. Neurourol Urodyn 2007; 26: 920-927.

22 Ikeda Y, Fry C, Hayashi F, Stolz D, Griffiths D, Kanai A. Role of gap junctions in spontaneous activity of the rat bladder. Am J Physiol Renal Physiol 2007; 293: F1018-F1025.

23 Ikeda Y, Kanai A. Urotheliogenic modulation of intrinsic activity in spinal cordtransected rat bladders: role of mucosal muscarinic receptors. Am J Physiol Renal Physiol 2008; 295: F454-F461.

24 Ikeda Y, Birder L, Buffington C, Roppolo J, Kanai A. Mucosal muscarinic receptors enhance bladder activity in cats with feline interstitial cystitis. J Urol 2009; 181: 1415-1422.

25 Neuhaus J, Pfeiffer F, Wolburg H, Horn LC, Dorschner W. Alterations in connexin expression in the bladder of patients with urge symptoms. BJU Int 2005; 96 670-676.

26 Birder L, Andersson KE. Urothelial signaling. Physiol Rev 2013; 93: 653-680.

27 Acharya P, Beckel J, Ruiz WG, Wang E, Rojas R, Birder L et al. Distribution of the tight junction proteins ZO-1, occludin, and claudin-4, -8 , and -12 in bladder epithelium. Am J Physiol Renal Physiol 2004; 287: F305-F318.

28 Janssen DA, Hoenderop JG, Jansen KC, Kemp AW, Heesakkers JP, Schalken JA. The mechanoreceptor TRPV4 is localized in adherence junctions of the human bladder urothelium: a morphological study. J Urol 2011; 186: 1121-1127.

29 Sant GR, Kempuraj D, Marchand JE, Theoharides TC. The mast cell in interstitial cystitis: role in pathophysiology and pathogenesis. Urology 2007; 69: 34-40.
30 Shie JH, Liu HT, Kuo HC. Increased cell apoptosis of urothelium mediated by inflammation in interstitial cystitis/painful bladder syndrome. Urology 2012; 79: 484 e7-484 e13.

31 Giannantoni A, Di Stasi SM, Nardicchi V, Zucchi A, Macchioni L, Bini V et al. Botulinum-A toxin injections into the detrusor muscle decrease nerve growth factor bladder tissue levels in patients with neurogenic detrusor overactivity. J Urol 2006; 175: 2341-2344.

32 Schurch B, Stohrer M, Kramer G, Schmid DM, Gaul G, Hauri D. Botulinum-A toxin for treating detrusor hyperreflexia in spinal cord injured patients: a new alternative to anticholinergic drugs? Preliminary results. J Urol 2000; 164: 692-697.

33 Kalsi V, Apostolidis A, Popat R, Gonzales G, Fowler CJ, Dasgupta P. Quality of life changes in patients with neurogenic versus idiopathic detrusor overactivity after intradetrusor injections of botulinum neurotoxin type A and correlations with lower urinary tract symptoms and urodynamic changes. Eur Urol 2006; 49: 528-535.

34 Karsenty G, Denys P, Amarenco G, De Seze M, Game X, Haab F et al. Botulinum toxin $A$ (Botox) intradetrusor injections in adults with neurogenic detrusor overactivity/ neurogenic overactive bladder: a systematic literature review. Eur Urol 2008; 53: 275-287.

35 Haferkamp A, Schurch B, Reitz A, Krengel U, Grosse J, Kramer G et al Lack of ultrastructural detrusor changes following endoscopic injection of botulinum toxin type a in overactive neurogenic bladder. Eur Urol 2004; 46: 784-791.

36 Rovner E, Dmochowski R, Chapple C, Thompson C, Lam W, Haag-Molkenteller C. OnabotulinumtoxinA improves urodynamic outcomes in patients with neurogenic detrusor overactivity. Neurourol Urodyn 2013; 32: 1109-1115.

37 Giannantoni A, Conte A, Farfariello V, Proietti S, Vianello A, Nardicchi V et al. Onabotulinumtoxin-A intradetrusorial injections modulate bladder expression of NGF, TrkA, p75 and TRPV1 in patients with detrusor overactivity. Pharmacol Res 2013; 68: $118-124$.

38 Collins VM, Daly DM, Liaskos M, McKay NG, Sellers D, Chapple C et al. OnabotulinumtoxinA significantly attenuates bladder afferent nerve firing and inhibits ATP release from the urothelium. BJU Int 2013; 112: 1018-1026.

39 Chen YC, Kuo HC. The therapeutic effects of repeated detrusor injections between 200 or 300 units of onabotulinumtoxinA in chronic spinal cord injured patients. Neurourol Urodyn 2014; 33: 129-134.

40 Chen CY, Liao CH, Kuo HC. Therapeutic effects of detrusor botulinum toxin A injection on neurogenic detrusor overactivity in patients with different levels of spinal cord injury and types of detrusor sphincter dyssynergia. Spinal Cord 2011; 49: 659-664.

41 Chuang FC, Kuo HC. Increased urothelial cell apoptosis and chronic inflammation are associated with recurrent urinary tract infection in women. PLOS ONE 2013; 8: e63760. 